\title{
Perfectly Regular and Perfectly Edge-Regular Intuitionistic Fuzzy Graphs
}

\author{
P. Syamala, K. R. Balasubramanian
}

\begin{abstract}
A Perfectly regular intuitionistic fuzzy graph is an intuitionistic fuzzy graph that is both regular and totally regular. In this paper we introduce and classify these types of intuitionistic fuzzy graphs and study several of their properties, including how two classes of intuitionistic fuzzy graphs structurally relate to one another and several of their spectral properties such as isospectral intuitionistic fuzzy graphs and when the energy of intuitionistic fuzzy graph is proportional to the energy of their underlying crisp graphs. These properties are studied in particular due to having at least one constant function $\mu$ and $\gamma$.
\end{abstract}

Keywords: Intuitionistic fuzzy graph, perfectly regular, perfectly edge regular intuitionistic fuzzy graph, Graph energy, Spectral intuitionistic fuzzy graph theory, Intuitionistic fuzzy matrix.

\section{INTRODUCTION}

Regular and totally regular fuzzy graphs were first introduced in [5]. The fuzzy edge analog of these concepts, edge regularity and total edge-regularity, were introduced and studied in [18]. These concepts of regularity for both vertices and edges in fuzzy graphs led to many advancements in the structural theory of fuzzy graphs. Several relevant marquee results stemming from this research include [1,3,4,6,8-17,19-23,25-30].

The purpose of this paper is to prepare for a study of those intuitionistic fuzzy graphs that concurrently exhibit both intuitionistic fuzzy vertex and edge-regular properties. These graphs will eventually help link certain intuitionistic fuzzy systems and crisp systems, allowing for greater case in computing properties of these fuzzy systems for modeling purposes [2] or optimizing these fuzzy networks [7]. We first study perfectly regular intuitionistic fuzzy graph which are both edge regular and totally edge-regular. Spectral properties of these classes of intuitionistic fuzzy graphs in particular will help relate notions of regularity in intuitionistic fuzzy graphs to crisp graphs, thus allowing for a deeper understanding of these special classes of intuitionistic fuzzy graphs.

Perfectly regular intuitionistic fuzzy graphs will be characterized in section 3 along with several initial results on perfectly regular intuitionistic fuzzy graphs. A similar study of perfectly edge-regular intuitionistic fuzzy graphs will be given in section 4 .

\section{Revised Manuscript Received on October 30, 2019.}

* Correspondence Author

Mrs. P.Syamala*, M. Phil Degree, St.Joseph's College, Hoshangabad, (M.P), India.

Dr. K.R.Bala Subramanian, M. Phil Degree, St.Joseph's College, Hoshangabad, (M.P), India.

(C) The Authors. Published by Blue Eyes Intelligence Engineering and BY-NC-ND license (http://creativecommons.org/licenses/by-nc-nd/4.0/) Sciences Publication (BEIESP). This is an open access article under the CC

From there, we will study the combination of these properties in intuitionistic fuzzy graphs by first studying the relationships between perfectly regular and perfectly edge regular intuitionistic fuzzy graphs in section 5 and then by providing a study of their adjacency matrices in section 6 . The intention of this body of work is to serve as the necessary preliminaries for the introduction and study of those intuitionistic fuzzy graphs which are both exhibiting concurrently constant function $\mu$ and $\gamma$ including those intuitionistic fuzzy graphs which are both perfectly regular and perfectly edge - regular. For an introduction to fuzzy graph theory and its basic definition, the reader is referred to [8]. For analysis notations and relevant limit theorems, the reader is referred to [24].

\section{PRELIMINARIES}

\section{Definition 2.1}

An intuitionistic fuzzy graph is of the form $G=(V, E)$ where, i) $\mathrm{V}=\left\{v_{1}, v_{2}, \ldots \ldots v_{n}\right\}$ such that $\mu_{1}: V \rightarrow[0,1]$ and $\gamma: V \rightarrow[0,1]$ denotes the degree of membership and non-membership of the element $v_{i} \in V$ respectively and

$0 \leq \mu_{1}\left(v_{1}\right)+\gamma_{1}\left(v_{1}\right) \leq 1 \forall v_{i} \mathrm{eV} \quad(i=1,2, \ldots . . n) \rightarrow(1)$

ii) $\mathrm{E} \subseteq V \times V$ where

$\mu_{2}: V \times V \rightarrow[0,1]$ and $\gamma_{2}: V \times V \rightarrow[0,1]$ are such that

$\mu_{2}\left(v_{1}, v_{2}\right) \leq \min \left\{\mu_{1}\left(v_{1}\right), \mu_{1}\left(v_{2}\right)\right\} \quad \rightarrow(2)$

$\gamma_{2}\left(v_{1}, v_{2}\right) \leq \max \left\{\gamma_{1}\left(v_{1}\right), \gamma_{1}\left(v_{2}\right)\right\} \quad \rightarrow(3)$

and

$0 \leq \mu_{2}\left(v_{1}, v_{2}\right)+\gamma_{1}\left(v_{1}, v_{2}\right) \leq 1 \forall\left(v_{1}, v_{2}\right) \in E(1,2, \ldots . . n)$

$\rightarrow(4)$

Definition 2.2

The degree of a vertex in an intuitionistic fuzzy graph is $d(v)$ $=\left(d_{\mu}(v), d_{\gamma}(v)\right)$ where

$$
\begin{aligned}
& d_{\mu}(v)=\sum_{u \neq v} \mu_{2}(u, v) \\
& d_{\gamma}(v)=\sum_{u \neq v} \gamma_{2}(u, v)
\end{aligned}
$$

\section{Definition 2.3}

The total degree of a vertex in an intuitionistic fuzzy graph is $\quad t d(v)=\left(t d_{\mu}(v), t d_{\gamma}(v)\right)$ Where

$$
\begin{aligned}
& t d_{\mu}(v)=d_{\mu}(v)+\mu_{1}(v) \\
& \text { and } t d_{\gamma}(v)=d_{\gamma}(v)+\gamma_{1}(v) .
\end{aligned}
$$

\section{Definition 2.4}

A regular intuitionistic fuzzy graph is an intuitionistic fuzzy graph with if all vertices have same degree.

\section{Definition 2.5}

A totally regular intuitionistic fuzzy graph is an intuitionistic fuzzy graph if all vertices have same total degree. 


\section{Perfectly Regular and Perfectly Edge-Regular Intuitionistic Fuzzy Graphs}

\section{Definition 2.6}

A perfectly regular intuitionistic fuzzy graph is an intuitionistic fuzzy graph that is both regular and totally regular.

\section{Definition 2.7}

The degree of an edge in an intuitionistic fuzzy graph is $d_{\mu}(u v)=d_{\mu}(u)+d_{\mu}(v)-2 \mu_{2}(u v)$ and $d_{\gamma}(u v)=d_{\gamma}(u)+d_{\gamma}(v)-2 \gamma_{2}(u v)$.

\section{Definition 2.8}

The total degree of an edge in an intuitionistic fuzzy graph is

$$
t d_{\mu}(u v)=d_{\mu}(u v)+\mu_{2}(u v) \text { and }
$$$$
t d_{\gamma}(u v)=d_{\gamma}(u v)+\gamma_{2}(u v) \text {. }
$$

\section{Definition 2.9}

An edge regular intuitionistic fuzzy graph is an intuitionistic fuzzy graph having

$$
d_{\mu}(u v)=k \text { and } d_{\gamma}(u v)=\mathrm{k} \forall u v \in E
$$

\section{Definition 3.0}

A perfectly edge-regular intuitionistic fuzzy graph is an intuitionistic fuzzy graph that is both edge-regular and totally edge-regular.

\section{Definition 3.11}

The order of an intuitionistic fuzzy graph is $o_{\mu}(\mathrm{G})=\sum_{v \in V} \mu_{1}(v)$ and $o_{\gamma}(\mathrm{G})=\sum_{v \in V} \gamma_{1}(v)$

\section{Definition 3.12}

An intuitionistic fuzzy graph is complete if $\mu_{2}(u v)=\mu_{1}(u) \Lambda \mu_{1}(v)$ and $\gamma_{2}(u v)=\gamma_{1}(u) \Lambda \gamma_{1}(v)$ $\forall u v \in E$.

\section{PERFECTLY REGULAR INTUITIONISTIC FUZZY GRAPH.} not totally regular and of totally regular intuitionistic fuzzy graph that are not regular. In that same work, Theorem 3.2 provided a necessary (but not sufficient) condition on the intuitionistic fuzzy subset $\gamma$ of $V \mu_{1}$ and $\gamma_{1}$ of $V$ for perfectly regular graphs, namely that $\mu_{1}: V \rightarrow[0,1]$ and $\gamma_{1}: V \rightarrow[0,1]$ must be a constant function.

\section{Theorem 3.1}

Let $\mathrm{G}=(V, \mu, \gamma)$ be a perfectly regular intuitionistic fuzzy graph. Then $\mu_{1}: V \rightarrow[0,1]$ and $\gamma_{1}: V \rightarrow[0,1]$ is a constant function.

\section{Proof}

Since $G$ is perfectly regular.

We have $\mathrm{G}$ is both $K_{1}$ regular and $K_{2}$ totally regular.

Then we have that,

$$
\begin{gathered}
\operatorname{td}(v)=\left[\left(d_{\mu}(v)+\mu_{1}(v)\right),\left(d_{\gamma}(v)+\gamma_{1}(v)\right)\right] \\
=\left[\left(d_{\mu}(u)+\mu_{1}(u)\right),\left(d_{\gamma}(u)+\gamma_{1}(u)\right)\right] \\
=\left[t d_{\mu}(u), t d_{\gamma}(u)\right] \forall u, v \in V
\end{gathered}
$$

Since, $\left[d_{\mu}(v), d_{\gamma}(v)\right]=\left[d_{\mu}(u), d_{\gamma}(u)\right]=K_{1}$ and $\left[t d_{\mu}(v), t d_{\gamma}(v)\right]=\left[t d_{\mu}(u), t d_{\gamma}(u)\right]=K_{2}$

We have that,

$$
\left(\mu_{1}(v), \gamma_{1}(v)\right)=\left(\mu_{1}(u), \gamma_{1}(u)\right)
$$

Hence if $\mathrm{G}$ is perfectly regular then $\mu_{1}$ and $\gamma_{1}$ must be a constant function.

\section{Theorem 3.2}

An intuitionistic fuzzy graph $\mathrm{G}=(V, \mu, \gamma)$ is perfectly regular if and only if it satisfies the following conditions

i) $\sum_{k \neq i}\left[\mu_{2}\left(v_{i} v_{k}\right), \gamma_{2}\left(v_{i} v_{k}\right)\right]$

$$
=\sum_{k \neq j}\left[\mu_{2}\left(v_{j} v_{k}\right), \gamma_{2}\left(v_{j} v_{k}\right)\right]
$$

Let $\mathrm{G}=(v, \mu, \gamma)$ be an intuitionistic fuzzy graphs that are

ii) $\left(\mu_{1}\left(v_{i}\right), \gamma_{1}\left(v_{i}\right)\right)=\left(\mu_{1}\left(v_{j}\right), \gamma_{1}\left(v_{j}\right)\right)$

\section{Proof}

$$
\forall i, j \forall \in\{1,2 \ldots . .|V|\}
$$

Let $\mathrm{G}$ be perfectly regular.

Be definition $\mathrm{G}$ is regular.

Hence it trivially satisfies (i).

Theorem 3.1 implies that condition (ii) is also met.

Conversely,

Let $G$ be an intuitionistic fuzzy graph satisfies both conditions (i) and (ii) $\left(\mu_{1}, \gamma_{1}\right)=\mathrm{c}$ is a constant function.

$\left[t d_{\mu}(v), t d_{\gamma}(v)\right]=k+c \quad \forall v e V$.

and thus $\mathrm{G}$ is both regular and totally regular.

Hence $G$ is perfectly regular.

Corollary 3.3

Let $G$ be a perfectly regular intuitionistic fuzzy graph.

Let $\left(\mu_{1}(v), \gamma_{1}(v)\right)=\mathrm{c} \forall v \in V$.

Then the order $\mathrm{G}$ is $\mathrm{O}(\mathrm{G})=\mathrm{c}|V|$.

\section{Theorem 3.4}

Let $\mathrm{G}$ be a perfectly regular intuitionistic fuzzy graph and Let $\left[d_{\mu}(v), d_{\gamma}(v)\right]=\mathrm{k} \forall v e V$.

Then the size of $\mathrm{G}$ is $\mathrm{S}(\mathrm{G})=\frac{k|v|}{2}$.

Proof

Since $\mathrm{G}$ is perfectly regular, we have that $\left(d_{\mu}(v), d_{\gamma}(v)\right)=\mathrm{k} \forall v e V$

Hence, $\sum_{v \in V}\left[d_{\mu}(v), d_{\gamma}(v)\right]=\mathrm{k}|v|$.

However, since $\left(d_{\mu}(v), d_{\gamma}(v)\right)=$

$\sum_{u \neq v}\left(\mu_{2}(u v), \gamma_{2}(u v)\right)$

We have that $\sum_{v \in V}\left[d_{\mu}(v), d_{\gamma}(v)\right]=$

$\sum_{v \in V} \sum_{u \neq v}\left(\mu_{2}(u v), \gamma_{2}(u v)\right)$

$$
\begin{aligned}
& =2 \sum_{u v \in E}\left(\mu_{2}(u v), \gamma_{2}(u v)\right) \\
& =2 \mathrm{~S}(\mathrm{G}) .
\end{aligned}
$$

Thus we conclude that the size of a perfectly regular intuitionistic fuzzy graph is $\frac{k(v)}{2}$.

\section{PERFECTLY EDGE - REGULAR INTUITIONISTIC FUZZY GRAPHS}

\section{Theorem 4.1}

Let $\mathrm{G}=(V, \mu, \gamma)$ be a perfectly edge-regular fuzzy graph. Then $\mu_{2}: V \times V \rightarrow[0,1]$ and $\gamma_{2}: V \times V \rightarrow[0,1]$ is a constant function.

\section{Proof}

Since $\mathrm{G}$ is perfectly edge -regular, We have that $\mathrm{G}$ is both $K_{1}$ edge regular and $K_{2}$ totally edge-regular.

Then we have that

$$
\begin{gathered}
K_{2}=t d(u v)=\left(d_{\mu}(u v)+\mu_{2}(u v)\right),\left(d_{\gamma}(u v)+\gamma_{2}(u v)\right) \\
=K_{1}+\left(\mu_{2}(u v), \gamma_{2}(u v)\right) \quad \rightarrow(1) \\
t d(x y)=\left[\left(\left(d_{\mu}(x y)+\mu_{2}(x y)\right),\left(d_{\gamma}(x y)+\gamma_{2}(x y)\right)\right]\right.
\end{gathered}
$$

Since $u v$ and $x y$ were arbitrarily choosen edges. Hence the proof. 


\section{Theorem 4.2}

An intuitionistic fuzzy graph $G=(V, \mu, V)$ is perfectly edge regular if and only if it satisfies the following conditions.

i) $\sum_{z \neq u}\left(\mu_{2}(u z), \gamma_{2}(u z)\right)+\sum_{z \neq v}\left(\mu_{2}(v z), \gamma_{2}(v z)\right)-2$ $\left(\mu_{2}(u v), \gamma_{2}(u v)\right)=\sum_{z \neq x}\left(\mu_{2}(x z), \gamma_{2}(x z)\right)+$ $\sum_{z \neq y}\left(\mu_{2}(y z), \gamma_{2}(y z)\right)-2\left(\mu_{2}(x y), \gamma_{2}(x y)\right)$ $\forall u v, x y \in E$

ii) $\left(\mu_{2}(u v), \gamma_{2}(u v)\right)=\left(\mu_{2}(x y), \gamma_{2}(x y)\right)$ $\forall u v, x y \in E$.

\section{Proof}

Since G is perfectly edge- regular and since (i) is the definition of an edge-regular intuitionistic fuzzy graph.

G obviously satisfies (i). By theorem 4.1, G also satisfies (ii)

Conversely,

Let $\mathrm{G}$ be an intuitionistic fuzzy graph satisfies both (i) and (ii) ie) $\mathrm{G}$ is $\mathrm{k}$ - edge regular and has a constant $\mu=c$. Since (i) is the definition of being edge-regular.

$$
\text { iii) }\left[t d_{\mu}(u v)+t d_{\gamma}(u v)\right]=k+c
$$

$=\left(t d_{\mu}(x y), t d_{\gamma}(x y)\right) \forall u v, x y \in E$.

\section{Corollary 4.3}

Let $\mathrm{G}$ be a perfectly edge regular intuitionistic fuzzy graph and let, $\left(\mu_{2}(u v), \gamma_{2}(u v)\right)=\mathrm{c} \forall u v \in E$. Then the size of $\mathrm{G}$ is $\mathrm{S}(\mathrm{G})=\mathrm{c}|E|$.

\section{Theorem 4.4}

Let $G$ be a perfectly edge regular intuitionistic fuzzy graph. Then the order $G$ is bounded between $\sum_{v \in V} \sum_{x \neq v}\left(\mu_{2}(x v), \gamma_{2}(x v) \leq O(G) \leq|v|\right.$.

\section{Proof}

As the upper bound is obvious, we need only to prove the lower bound.

This follows directly from the definition of $\left(\mu_{2}(u v), \gamma_{2}(u v)\right) \leq\left(\mu_{1}(u), \gamma_{1}(u) \Lambda\left(\mu_{1}(v), \gamma_{1}(v)\right)\right.$

Thus we have that $\left(\mu_{1}(v), \gamma_{1}(v)\right) \geq \sum_{x \neq v} V\left(\mu_{2}(x v), \gamma_{2}(x v)\right)$ is a lower bound for $\left(\mu_{1}(v), \gamma_{1}(v) \forall v e V\right.$.

The sum of these individual lower bounds for $\left(\mu_{1}(v), \gamma_{1}(v)\right)$ is precisely the lower bound stated for $\mathrm{O}(\mathrm{G})$ in the theorem.

\section{Theorem 4.5}

Let $\mathrm{G}$ be a perfectly edge - regular complete intuitionistic fuzzy graph and let $u \in V$ if there exists $v \in N(u) \ni$ : $\left(\mu_{1}(u), \gamma_{1}(u)\right) \leq\left(\mu_{1}(v), \gamma_{1}(v)\right)$. Then there exists an edge uveE $\ni:\left(\mu_{2}(u v), \gamma_{2}(u v)\right)=\left(\mu_{1}(u), \gamma_{1}(u)\right)$.

\section{Proof}

Since G is complete.

$\left(\left(\mu_{2}(u v), \gamma_{2}(u v)\right)=\left(\mu_{1}(u), \gamma_{1}(u)\right) \Lambda\left(\mu_{1}(v), \gamma_{1}(v)\right)\right.$ choose $v \ni v \in N(u)$ and $\left(\mu_{1}(v), \gamma_{1}(v)\right) \geq\left(\mu_{1}(u), \gamma_{1}(u)\right)$ Then $\left(\mu_{2}(u v), \gamma_{2}(u v)\right)=\left(\mu_{1}(u), \gamma_{1}(u)\right)$.

\section{Theorem 4.6}

Let $P_{u}$ be an ordering of the $v \in N(u)$ such that $x \leq y=$ $\left(\mu_{1}(x), \gamma_{1}(x)\right) \leq\left(\mu_{1}(y), \gamma_{1}(y)\right)$. If $P_{u}$ does not have a unique greatest element for all $u \in V$, then $\sum_{v \in V} \Lambda_{x \neq v}$ $\left(\mu_{2}(x v), \gamma_{2}(x v)=\mathrm{O}(\mathrm{G})\right.$.

\section{Proof}

Since $\mathrm{G}$ is complete and since there is no unique greatest element in $P_{u} \forall u \in V$.

We have that $\forall v \in V$ there exists $u \in V \quad \exists$ $\left(\mu_{1}(v), \gamma_{1}(v)\right) \leq\left(\mu_{1}(v), \gamma_{1}(v)\right)$.

We have that

$$
\sum_{v \in V} \wedge_{x \neq v}\left(\mu_{2}(x v), \gamma_{2}(x v)=\mathrm{O}(\mathrm{G})\right.
$$

\section{Theorem 4.7}

Hence the proof.

Let $O(G)$ represent the order of a perfectly edge-regular complete intuitionistic fuzzy graph $\mathrm{G}$ with constant $\left(\mu_{2}, \gamma_{2}\right)=$ c. Let $\mathrm{T}$ be the independent set of all vertices $v \ni u v \in E \ni\left(\mu_{2}(u v), \gamma_{2}(u v)\right)=\left(\left(\mu_{1}(v), \gamma_{1}(v)\right)\right.$ and let $|T|=\alpha|V| \quad$.Then (1- $\alpha(1-c) \quad) \quad \mathrm{O}(\mathrm{G})$ $\leq \sum_{v \in V} \wedge_{x \neq V}\left(\mu_{2}(x v), \gamma_{2}(x v)\right) \leq O(G)$.

Proof

As the upper bound was established.

We need only to prove the lower bound.

Let $V_{1}$ be the set of vertices $v e V \ni u v \in E \ni\left(\mu_{2}(u v), \gamma_{2}(u v)\right)=\left(\mu_{1}(v), \gamma_{1}(v)\right)$ and let $V_{2}=\mathrm{V} \backslash V_{1}$.

ie) $V_{2}$ is the independent set described in the statement of the theorem.

Hence $|V|=\alpha|V|$.

Thus we may write a lower bound for $O$ (G) as $\sum_{v \in V_{1}} \wedge_{x \neq v}\left(\mu_{2}(x v), \gamma_{2}(x v)\right)+\sum_{v \in V_{2}} \wedge_{x \neq v}\left(\mu_{2}(x v), \gamma_{2}(x v)\right)$ $\leq \sum_{v \in V_{1}}\left(\mu_{1}(v), \gamma_{1}(v)\right)+\sum_{v \in v_{2}}\left(\mu_{1}(v), \gamma_{1}(v)\right) \rightarrow(1)$

By Definition of $V_{1}$.

We have that $\sum_{v \in V_{1}} \wedge_{x \neq v}\left(\mu_{2}(x v), \gamma_{2}(x v)\right)=$ $\sum_{v \in V_{1}}\left(\mu_{1}(v), \gamma_{1}(v)\right)$

So we may rewrite this lower bound as

$\sum_{v \in V_{1}} \wedge_{x \neq v}\left(\mu_{2}(x v), \gamma_{2}(x v)\right) \leq \sum_{v \in V_{2}}\left(\mu_{1}(v), \gamma_{1}(v)\right)$ with equality holding only when $V_{2}=\varphi$.

Since $\left(\mu_{2}, \gamma_{2}\right)=\mathrm{c}$ is a constant function due to the perfect edge-regularity of $\mathrm{G}$.

We may obtain an upper bound on the difference between the two sides of our inequality as

$\alpha(1-c)|V| \quad \geq \sum_{v \in V_{2}}\left(\mu_{1}(v) \quad, \quad \gamma_{1}(v) \quad\right)$

$\sum_{v \in V_{2}} \wedge_{x \neq v}\left(\mu_{2}(x v), \gamma_{2}(x v)\right) \quad \rightarrow(2)$

Thus we may bound $\sum_{v \in V} \wedge_{x \neq v}\left(\mu_{2}(x v), \gamma_{2}(x v)\right)$ in both direction as follows

$\sum_{v \in V}\left(\mu_{1}(v), \gamma_{1}(v)\right)-\alpha(1-c)|V| \leq$

$\sum_{v \in V} \wedge_{x \neq v}\left(\mu_{2}(x v), \gamma_{2}(x v)\right)$

$\leq \sum_{v \in V}\left(\mu_{1}(v), \gamma_{1}(v)\right)$

By dividing through by $\mathrm{O}(\mathrm{G})$ we obtain

$$
1-\frac{\alpha(1-c)|V|}{O(G)} \leq \frac{\sum_{v e V} \wedge_{x \neq v\left(\mu_{2}(x v), \gamma_{2}(x v)\right.}}{O(G)} \leq 1 \rightarrow(4)
$$

By noticing that $1 \leq \frac{|V|}{O(G)} \leq \frac{1}{C}$.

and making a small sacrifice to lower bound, we obtain $1-\alpha(1-c) \leq \frac{\sum_{v e V} \Lambda_{x \neq v}\left(\mu_{2}(x v), \gamma_{2}(x v)\right)}{O(G)} \leq 1 \quad \rightarrow(5)$

By multiplying through by $\mathrm{O}(\mathrm{G})$

Hence the proof.

\section{V.RELATING VERTEX AND EDGE -REGULARITY IN INTUITIONISTIC FUZZY GRAPHS}

In this section we study what additional properties are needed for one form of regularity in intuitionistic fuzzy graphs to imply the other form of regularity. A perfectly regular intuitionistic fuzzy graphs that is regular and has a constant function $\sigma$.

\section{Theorem 5.1}

If $\mathrm{G}$ is regular and $\mu=c$ is a constant function then $\mathrm{G}$ is perfectly edge - regular. 


\section{Perfectly Regular and Perfectly Edge-Regular Intuitionistic Fuzzy Graphs}

\section{Proof}

Let $\mathrm{G}$ be a regular intuitionistic fuzzy graph and let $\mu=c$ be a constant function. Then

$\left(d_{\mu}(v), d_{\gamma}(v)\right)=\mathrm{k} \forall v \in V$.

Where $\mathrm{k}$ is simply a multiple of c. The degree of an arbitrary edge of $\mathrm{G}$ is then

$\left(d_{\mu}(u v), d_{\gamma}(u v)\right)=\left(d_{\mu}(u), d_{\gamma}(u)\right)+\left(d_{\mu}(v), d_{\gamma}(v)\right)-$ $2\left(\mu_{2}(u v), \gamma_{2}(u v)\right)=2 \mathrm{k}-2 \mathrm{c}=2(\mathrm{k}-\mathrm{c})$

Hence $\mathrm{G}$ is edge - regular.

Since the total degree of an edge in an intuitionistic fuzzy graph is

$\left(t d_{\mu}(u v)+t d_{\gamma}(u v)\right)=$

$$
\left(d_{\mu}(u v), d_{\gamma}(u v)\right)+\left(\mu_{2}(u v), \gamma_{2}(u v)\right)
$$

We have,

$\left(t d_{\mu}(u v)+t d_{\gamma}(u v)\right)=2(\mathrm{k}-\mathrm{c})+\mathrm{c}=2 \mathrm{k}-\mathrm{c}$ for all edges in G.

Thus $\mathrm{G}$ is totally edge-regular and therefore perfectly edge-regular.

\section{Example 5.2}

Consider figure is totally regular and not a regular and cannot be totally edge - regular as it is not edge regular.
$(0.2,0.2) \mathrm{b}$

$(0.1,0.1)$

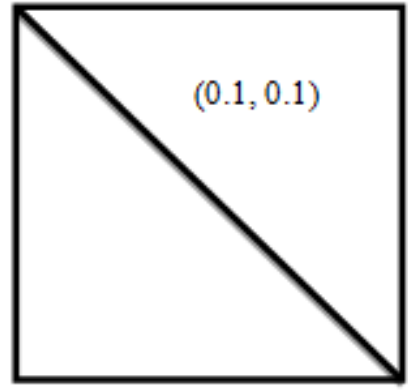

c $(0.2,0.2)$

$(0.1,0.1)$

$\left(\mu_{1}(a), \gamma_{1}(a)\right)=\left(\mu_{1}(c), \gamma_{1}(c)\right)=(0.2,0.2)$

$\left(\mu_{1}(b), \gamma_{1}(b)\right)=\left(\mu_{1}(d), \gamma_{1}(d)\right)=(0.1,0.1)$

and $\left(\mu_{2}(a b), \gamma_{2}(a b)\right)=\left(\mu_{2}(b c), \gamma_{2}(b c)\right)=(0.1,0.1)$ $\left(\mu_{2}(c d), \gamma_{2}(c d)\right)=\left(\mu_{2}(a d), \gamma_{2}(a d)\right)=\left(\mu_{2}(b d), \gamma_{2}(b d)\right)=$ $(0.1,0.1)$.

While the degree of $b$ and $d$ is $(0.3,0.3)$.

The total degree of every vertex is $(0.4,0.4)$.

Hence,

A an constant $\left(\mu_{2}, \gamma_{2}\right)$ and is totally regular but is not regular.

To see that it is not edge - regular.

Consider the edges ab and bd.

We have,

$\left(d_{\mu}(a b), d_{\gamma}(a b)\right)=0.3 \neq 0.4=\left(d_{\mu}(b d), d_{\gamma}(b d)\right)$

Since $\mu$ is constant.

Clearly, this figure cannot be totally edge-regular as it is not edge-regular.

Theorem 5.3

If $G$ is perfectly regular and complete, then $G$ is perfectly edge - regular

Proof

Since $\mathrm{G}$ is perfectly regular.

$\left.\mu_{1}(u), \gamma_{1}(u)\right)=\left(\mu_{1}(v), \gamma_{1}(v)\right)$

Since $\mathrm{G}$ is complete.

$\left(\mu_{2}(u v), \gamma_{2}(u v)\right)=\left(\mu_{1}(u), \gamma_{1}(u)\right)$

$$
\begin{gathered}
\Lambda\left(\mu_{1}(v), \gamma_{1}(v)\right) \\
\forall u v \in E
\end{gathered}
$$

(1) and (2) $\Rightarrow\left(\mu_{2}, \gamma_{2}\right)$ is a constant function.

Hence the Proof.

\section{SOME SPECTRAL PROPERTIES OF EDGE-REGULAR INTUITIONISTIC FUZZY GRAPH}

In this section we study some basic spectral properties of perfectly edge -regular intuitionistic fuzzy graphs. The adjacency matrix of an intuitionistic fuzzy graph $A(G)$ is defined as $\mathrm{A}(\mathrm{G})=\left(a_{i j}\right)=\left(\mu_{2}\left(v_{i} v_{j}\right), \gamma_{2}\left(v_{i} v_{j}\right)\right)$.

\section{Remark 6.1}

Let $\mathrm{G}$ be a perfectly edge-regular intuitionistic fuzzy graph with $\left(\mu_{2}, \gamma_{2}\right)=\mathrm{c}$ and let $G^{*}$ be the underlying crisp graph of G.Then $\mathrm{A}(\mathrm{G})=\mathrm{c} \mathrm{A}\left(G^{*}\right)$

\section{Theorem 6.2}

Let $G$ be a perfectly edge- regular fuzzy graph and let $G^{*}$ be its underlying crisp graph. If $\lambda$ is an eigen value of $G$ then $c \lambda$ is an eigen value of $\mathrm{G}$.

\section{Proof}

From 6.2 we have that the multiplicities of the eigenvalue from will be unchanged and that the eigenvalues of the intuitionistic fuzzy graph will both scaled by c,

Hence $G_{1}$ and $G_{2}$ are isospectral

Theorem 6.3

Let $G_{1}$ and $G_{2}$ be two perfectly edge-regular intuitionistic fuzzy graphs with respective underlying crisp graphs $G_{1}{ }^{*}$ and $G_{2}{ }^{*}$ respectively. If $G_{1}{ }^{*}$ and $G_{2}{ }^{*}$ are isospectral and $c_{1}=c_{2}$ then $G_{1}$ and $G_{2}$ are isospectral.

\section{Theorem 6.4}

Let $\mathrm{G}$ be a perfectly edge-regular intuitionistic fuzzy graph and let $G^{*}$ be its underlying crisp graph. If the energy of $G^{*}$ is $E(G)$ then the energy of $G$ is $E(G)=c E\left(G^{*}\right)$.

\section{Proof}

By definition the energy of $G^{*}$ given by $\mathrm{E}(\mathrm{G})=\sum_{i=1}^{n}\left|\lambda_{i}\right|$ where the $\lambda_{i}$ are the eigen values of $G^{*}$.

From theorem 6.2 We have that energy of $\mathrm{G}$ is given by $\mathrm{E}(\mathrm{G})=\sum_{i=1}^{n}\left|\mathrm{c} \lambda_{i}\right|=\mathrm{c}^{\sum_{i=1}^{n}\left|\lambda_{i}\right|}=\mathrm{cE}\left(G^{*}\right)$.

\section{CONCLUSION}

In this paper, we began a systematic study of two classes of intuitionistic fuzzy graph perfectly regular and perfectly edge-regular of intuitionistic fuzzy graphs that link intuitionistic fuzzy graph theory to fuzzy graph in several important aspects, most notably by studying some of the structural and spectral properties of these intuitionistic graphs.

\section{REFERENCES}

1. 1. M. Akram and W.A. Dudek, Regular bipolar fuzzy graphs, Neural Computing and Applications, 21(1)(2012) 197-205.

2. S. Elizabeth and L. Sujatha, Medical diagnosis based on interval valued fuzzy number matrices, Annals of Pure and Applied Mathematics, 7(1)(2014) 91-96. 
3. A. Nagoor Gani and M. Basheer Ahamed, Order and size in fuzzy graphs, Bulletin of Pure and Applied Sciences, 22(1)(2003) 145-148.

4. A. Nagoor Gani and S.R. Latha, On irregular fuzzy graphs, Applie d Mathematical Sciences, 6(11)(2012) 517-523.

5. A. Nagoor Gani and K. Radha, On regular fuzzy graphs, Journal of Physical Sciences, 12(2008) 33-40.

6. A. Kishore and M.S. Sunitha, Strong chromatic number of fuzzy graphs, Annals of Pure and Applied Mathematics, 7(2)(2014) 52-60.

7. L'aszl'o T K'oczy, Fuzzy graphs in the evaluation and optimization of networks, Fuzzy sets and systems, 46(3)(1992) 307-319.

8. N.R. Santhi Maheswari and C. Sekar, On m-neighbourly irregular fuzzy graphs, International Journal of Mathematics and Soft Computing, 5(2)(2015) 145-153.

9. N.R. Santhi Maheswari and C. Sekar, On (m , k) - regular fuzzy graphs, International Journal of Mathematical Archive, 7(1)(2016) 1-7.

10. N.R. Santhi Maheswari and C. Sekar, On Pseudo regular fuzzy graphs, Annals of Pure and Applied Mathematics, 11(1)(2016) 105-113.

11. S. Maheswari and C. Sekar, On $(2, k)$ - regular and totally $(2, k)$ regular fuzzy graphs, International Journal of Mathematics and Soft Computing, 4(2)(2014) 59-69.

12. J. Manora and I. Paulraj Jayasimman, Neighborhood sets and neighborhood polynomial of cycles, Annals of Pure and Applied Mathematics, 7(2)(2014) 45-51.

13. S.P. Nandhini and E. Nandhini, Strongly irregular fuzzy graphs, International Journal of Mathematical Archive, 5(5)(2014) 110-114

14. S. Ravi Narayanan and N.R. Santhi Maheswari, Highly totally irregular fuzzy graphs, Journal of Computer and Mathematical Sciences, 4(6)(2013) 403-459.

15. M. Pal and H. Rashmanlou, Irregular interval- valued fuzzy graphs, Annals of Pure and Applied Mathematics, 3(1)(2013) 56-66.

16. K. Radha and N. Kumaravel, On edge regular fuzzy graphs, International Journal of Mathematical Archive, 5(9)(2014) 100-112.

17. K. Radha and N. Kumaravel, On edge regular bipolar fuzzy graphs, Annals of Pure and Applied Mathematics, 10(2)(2015) 129-139.

18. K. Radha and N. Kumaravel, The edge degree and the edge regular properties of truncations of fuzzy graphs, Bulletin of Mathematics and Statistics Research,4(3)(2016) 7-16.

19. K.Radha and N.Kumaravel, On edge regular square fuzzy graphs, International Journal of Applied Mathematical Sciences, 9(2),(2016)169-176.

20. 20.K.Radha and M.Vijaya, Totally regular property of the join of two fuzzy graphs, International Journal of Fuzzy Mathematical Archive, 8(1)(2015) 9-17.

21. P.Rajarajeswari and P.Dhanalakshmi, Interval valued fuzzy soft matrix theory, Annals of pure and Applied Mathematics, 7(2)(2014) 61-72

22. 22.S.Samanta and M.Pal, Irregular bipolar fuzzy graphs, International Journal of Applications of Fuzzy Sets, 2(2012) 91-102

23. S. Samanta, B. Sarkar, D. Shin, M. Pal, Completeness and regularity of generalized fuzzy graphs, Springer plus, 5(1)(2016) 1979.

24. S. Samanta and M.Pal, Fuzzy k-competition graphs and p- competition fuzzy graphs, Fuzzy Inf.Eng., 5(2)(2013) 191-204.

25. A. Edward Samuel and C. Kayalvizhi, On k-regular chromatic fuzzy graphs, Advances in Fuzzy sets and systems, 19(2)(2015) 155.

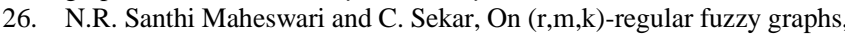
Mathematical Combinatorics, 1(2016) 18-26.

27. N.R. Santhi Maheswari and C. Sekar, On strongly edge irregular fuzzy graphs, Kragujevac Journal of Mathematics, 40(1)(2016) 125-135.

28. S. Sebastian and Ann Mary Philip, Regular and interval valued fuzzy graphs, Journal of Computer and Mathematical Sciences, 8(7)(2017) 309-322.

29. M.S. Sunitha and S. Mathew, Fuzzy graph theory: A survey, Annals of Pure and Applied Mathematics, 4(1)(2013) 92-110.

\section{AUTHORS PROFILE}

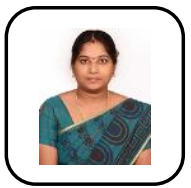

Mrs.P.Syamala received M.Phil degree from

St.Joseph's College, Trichy in the year 2011. She has 5 years and 11 months of experience in teaching. Now she is doing research in Intuitionistic Fuzzy Graphs.

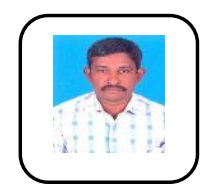

Dr.K.R.Bala Subramanian recieved Ph.D. degree in the year 2013. His field of specialization is Fuzzy Algebra and its applications. He has published 25 papers in National and International journals. He has 17 years of experience in teaching. 